\title{
Niche Cells and Signals that Regulate Lung Alveolar Stem Cells In Vivo
}

\author{
Nicholas H. Juul, ${ }^{1,2}$ Courtney A. Stockman, ${ }^{2}$ and Tushar J. Desai ${ }^{1,2}$ \\ ${ }^{1}$ Department of Medicine, Division of Pulmonary, Allergy \& Critical Care; ${ }^{2}$ Institute for Stem Cell Biology \\ \& Regenerative Medicine, Stanford University School of Medicine, Stanford, California 94305, USA \\ Correspondence: tdesai@stanford.edu
}

\begin{abstract}
The distal lung is a honeycomb-like collection of delicate gas exchange sacs called alveoli lined by two interspersed epithelial cell types: the cuboidal, surfactant-producing alveolar type II (AT2) and the flat, gas-exchanging alveolar type I (AT1) cell. During aging, a subset of AT2 cells expressing the canonical Wnt target gene, Axin2, function as stem cells, renewing themselves while generating new AT1 and AT2 cells. Wnt activity endows AT2 cells with proliferative competency, enabling them to respond to activating cues, and simultaneously blocks AT2 to AT1 cell transdifferentiation. Acute alveolar injury rapidly expands the AT2 stem cell pool by transiently inducing Wnt signaling activity in "bulk" AT2 cells, facilitating rapid epithelial repair. AT2 cell "stemness" is thus tightly regulated by access to Wnts, supplied by a specialized single-cell fibroblast niche during maintenance and by AT2 cells themselves during injury repair. Two non-AT2 "reserve" cell populations residing in the distal airways also contribute to alveolar repair, but only after widespread epithelial injury, when they rapidly proliferate, migrate, and differentiate into airway and alveolar lineages. Here, we review alveolar renewal and repair with a focus on the niches, rather than the stem cells, highlighting what is known about the cellular and molecular mechanisms by which they control stem cell activity in vivo.
\end{abstract}

The he skin, gastrointestinal (GI) tract and lung are all large epithelial surfaces of the body that are constantly exposed to the environment. Unlike the skin and GI tract, however, the lung is characterized by slow cellular renewal (Spencer and Shorter 1962; Evans and Bils 1969). Continuous turnover of the skin and GI epithelium is accomplished by dedicated stem cells residing in physically sheltered niches. This anatomical sequestration of stem cells may be protective against the development of cancer, because their progeny that will be the first to encounter mutagenic toxins are destined to be rapidly extruded. The alveolar epithelium, in contrast, undergoes slow turnover during aging by intermittent activation of a small subset of "bifunctional" alveolar epithelial type II (AT2) stem cells, socalled because they constitutively execute both physiologic and regenerative functions (Logan and Desai 2015). Unlike stem cells for the skin and gut, the AT2 stem cells that renew alveoli during aging are not physically shielded from

Editors: Cristina Lo Celso, Kristy Red-Horse, and Fiona M. Watt

Additional Perspectives on Stem Cells: From Biological Principles to Regenerative Medicine available at www.cshperspectives.org

Copyright (C) 2020 Cold Spring Harbor Laboratory Press; all rights reserved; doi: 10.1101/cshperspect.a035717

Cite this article as Cold Spring Harb Perspect Biol 2020;12:a035717 
N.H. Juul et al.

the environment; however, they make up a small proportion of the overall epithelial population (Desai et al. 2014). Although maintaining a small number of AT2 stem cells limits the opportunities for toxin-induced oncogenic transformation, it also carries the risk of inadequate regenerative reserve for surviving acute alveolar injury. The lung has evolved an elegant solution to this existential threat, by rapidly harnessing "bulk" AT2 cells to transiently participate in alveolar repair (Evans et al. 1973; Adamson and Bowden 1974). Two non-AT2 "reserve" alveolar progenitors, so-called because they are only induced following major lung injury, reside in the distalmost bronchioles and can regenerate both airway and alveolar epithelial cells. These facultative populations have been identified by using a variety of acute lung injury models, including ablation of specific epithelial cell types (diphtheria toxin, naphthalene); more generalized cell killing (hyperoxia, bleomycin, acid instillation); infection (H1N1 influenza or bacterial pneumonia); and stimulation of compensatory lung regrowth (unilateral pneumonectomy, refeeding after severe caloric restriction). Despite fundamental differences between these models (e.g., infectious vs. noninfectious, diffuse vs. alveolarrestricted injury), some generalizable principles of alveolar progenitor regulation have emerged.

Our most comprehensive understanding of progenitor regulation at present is of the AT2 stem cell, for which it is apparent that the niche controls stem cell number, proliferative competency, and differentiation outcome. However, substantial progress has recently been made in mapping the activity of the "reserve" progenitors by combining in vivo lineage tracing with experimental lung injury. Although the cellular niches harboring non-AT2 "reserve" alveolar progenitors have not yet been delineated, targeted gene deletion followed by induction of acute injury has revealed some of the signals important for their activity. Here, we summarize the available literature to present a "niche-centric" view of these distinct stem and progenitor cell populations. Our review includes what is known and outstanding about how they are controlled in health and perspectives on how their dysregulation may contribute to alveolar disease.

\section{IDENTITIES, LOCATIONS, AND BEHAVIORS OF CELLS THAT RENEW AND REPAIR PULMONARY ALVEOLI}

\section{Anatomical Compartments and Cellular Microenvironments Harboring Alveolar Progenitors}

There are at least three distinct cell populations that have been shown by in vivo fate mapping to generate new AT2 and AT1 cells (see Figs. 1 and 2, Legend panel). The originally identified and most studied is the AT2 cell (Kapanci et al. 1969; Adamson and Bowden 1974; Barkauskas et al. 2013; Desai et al. 2014), which also plays a critical physiological role in producing surfactant. AT2 cells express surfactant-associated protein C $(S f t p C)$ and are restricted to the distal gas-exchange (alveolar) region of the lung, where they are interspersed among flat AT1 cells in a monolayer epithelial sheet (Low 1953; Bertalanffy and Leblond 1955). In rat, AT2 cells have been estimated to be from 1.5 to 2 times as prevalent as AT1 cells, but the latter comprise $95 \%$ or more of the alveolar surface area because of their expansive morphology (Haies et al. 1981; Crapo et al. 1983). Although only a specialized subset of AT2 cells appears to act as stem cells during maintenance, "bulk" AT2 cells become transiently activated following acute alveolar injury, as described above.

A second population was originally shown to exhibit stem cell activity in vitro, capable of generating both alveolar and bronchiolar epithelial lineages in spheroid culture. This class, originally referred to as the bronchioalveolar stem cell (BASC) and now, as the $\mathrm{EPCAM}^{+} /$ $\mathrm{SCA}^{+}{ }^{+}$stem cell, coexpresses the AT2 marker $S f t p C$ and the club cell marker secretoglobin 1A1 (Scgb1a1) and resides at the end of the terminal bronchiole. It is estimated that between $25 \%$ and $50 \%$ of terminal bronchioles harbor at least one BASC (Kim et al. 2005). This Sftpc and Scgb1a1 double-positive population was recently specifically marked and lineage-traced in vivo and shown to contribute locally to both airway and alveolar lineages following severe acute lung injury (Liu et al. 2019).

A third alveolar progenitor class also resides outside the gas exchange compartment, in 


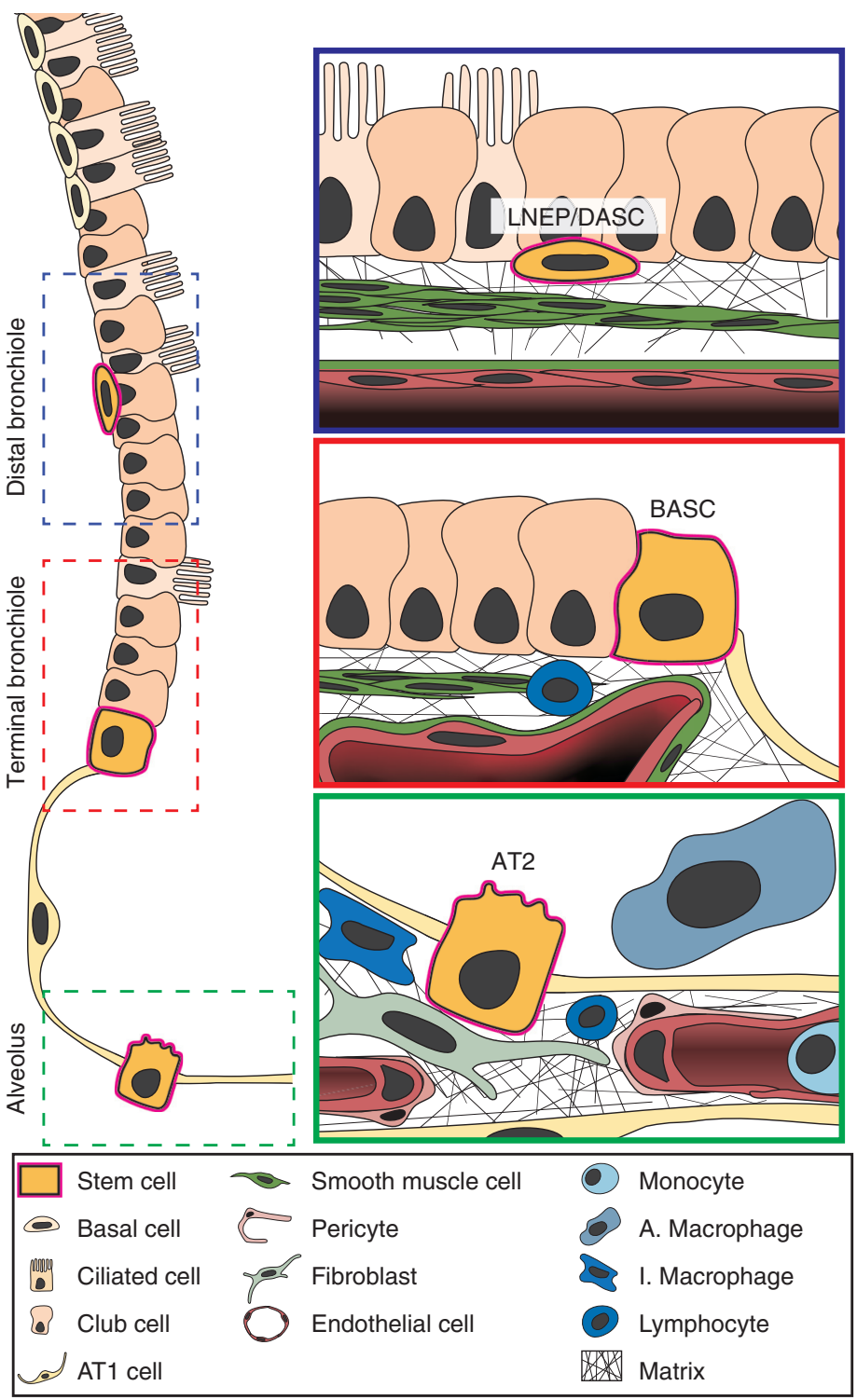

Figure 1. Anatomic domains and cellular microenvironments for alveolar progenitor cells. The three bestcharacterized alveolar stem and progenitor cells are schematized within the distal lung (left) with a close-up of the microenvironment in which each resides (right). (AT2) Alveolar epithelial type II cell, (BASC) bronchioalveolar stem cell, (LNEP/DASC) lineage-negative epithelial progenitor/distal airway stem cell, (A) alveolar, (I) interstitial.

slightly more proximal bronchioles than the BASC. These progenitors were identified by two different research groups, and although it is not entirely clear whether they are the same population, we will consider them as such because they are similar in anatomical localization, marker expression, and behavior (Kumar et al. 2011; Vaughan et al. 2015; Zuo et al. 2015). The lineage-negative epithelial progenitor (LNEP)/ distal airway stem cell (DASC) comprises a minor population in distal bronchioles that resemble basal cells in morphology and by expression 
N.H. Juul et al.
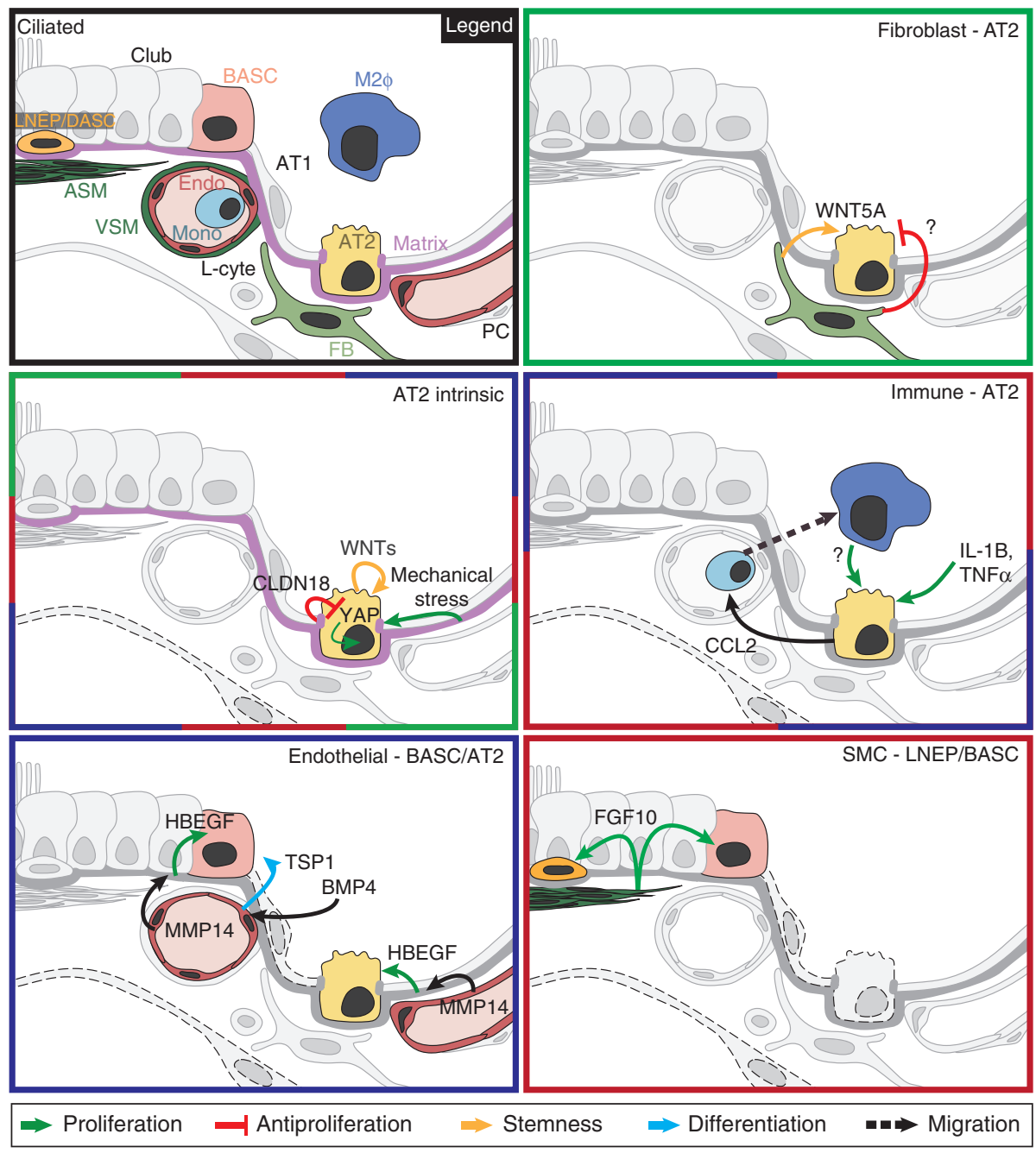

Figure 2. Signaling between alveolar stem and niche cells operative in specific experimental perturbations and contexts. (Top left) Panel summarizes each color-coded cell type and its position relative to the stem cells. Each additional panel schematizes the molecular signals and their sources that impact on the stem cell. The arrows are coded to indicate specific effects on the stem cell (legend below the panels), and the box outlining each panel is color-coded to indicate the experimental context in which the signaling is operative (maintenance; repair; pneumonectomy). (AT1) Alveolar epithelial type I, (AT2) alveolar epithelial type II, (BASC) bronchioalveolar stem cell, (Endo) endothelial cell, (FB) fibroblast, (LNEP/DASC) lineage-negative epithelial progenitor/distal airway stem cell, (L-cyte) lymphocyte, (AM) alveolar macrophage, (M2) M2 polarized macrophage, (Mono) monocyte, (PC) pericyte, (ASM) airway smooth muscle cell, (VSM) vascular smooth muscle, (?) unknown signal(s).

of keratin 5 (Krt5). Like basal stem cells that reside in more proximal airways, LNEP/DASCs can replenish club and ciliated lineages, but unlike basal cells they have also been shown to generate new AT2 cells (Rock et al. 2009). LNEP/ DASC cells have not been comprehensively mapped, but on average there is one LNEP per three small airways (Vaughan et al. 2015). Following widespread lung injury, LNEP/DASC cells extensively proliferate and spread into the alveolar compartment. Once there, a subset integrates into the epithelial monolayer, differen- 
tiating into AT2 cells that can go on to generate new AT1 cells. The remainder curiously fail to either integrate or differentiate, remaining physically clustered as distinctive "pods" of cells that continue to express Krt5 and presumably do not contribute to gas exchange (Ray et al. 2016).

AT2 Cells Are the Primary Stem Cells in Steady State and during Aging, whereas Non-AT2

Alveolar Progenitors Are Activated by Widespread Epithelial Injury

Broad genetic marking of the AT2 cell population followed by a long-term "chase" revealed that the proportion of labeled AT2 cells did not dilute over time, suggesting that mouse AT2 cells replenish themselves in adult life and during aging (Desai et al. 2014). This result implies that BASCs and LNEP/DASCs do not generate new AT2 cells in the absence of injury, which is corroborated by lineage-tracing experiments showing their lack of contribution to alveolar maintenance (Rawlins et al. 2009; Vaughan et al. 2015; Liu et al. 2019). After recovery from severe lung injury involving endotracheal bleomycin administration or H1N1 influenza pneumonia, however, the proportion of marked AT2 cells was reduced (Chapman et al. 2011). Because AT2 cells were broadly tagged before the injury, most unmarked AT2 cells observed after recovery presumably derived from non-AT2 cells. Candidate non-AT2 alveolar progenitors include BASCs, LNEP/DASCs, a minor population of integrin $\alpha 6 \beta 4^{+}$alveolar epithelial cells, and AT1 cells (Chapman et al. 2011; Jain et al. 2015). Lineage-tracing supports the contribution of each of these classes to new alveolar cells except for the integrin $\alpha 6 \beta 4^{+}$alveolar population, which has not yet been specifically marked and lineage traced in vivo.

BASCs have been shown to contribute to a modest number of cells in alveoli bordering terminal bronchioles following severe lung injury, whereas LNEP/DASCs massively expand and infiltrate the alveolar compartment. During their proliferation and distal spread, LNEP/ DASC cells appear to maintain their basal cell-like phenotype, with a subset giving rise to new club and ciliated cells along the way.
Although some progeny are found to have generated AT2 cells, others remain physically clustered as nonphysiologic "keratin pods" described above. Whether or not LNEP/DASCs give rise to BASCs or vice versa following severe injuries involving the distal airways has not yet been specifically examined.

\section{THE AT2 STEM CELL NICHE IS THE ALVEOLAR AGING NICHE}

The AT2 Stem Cell-Fibroblast Connection: A Molecular Basis for an Intimate Physical Association

In the 1950s, when the emergence of electron microscopy (EM) enabled visualization of alveolar architecture at subcellular resolution, researchers noted that some AT2 cells appeared to physically contact underlying fibroblasts via protrusions across the intervening basal lamina (Sirianni et al. 2003). By ultrastructure, there appeared to be at least three distinct alveolar fibroblast classes. One of these, the lipofibroblast, was typically juxtaposed to AT2 cells and believed to supply them with lipid for the synthesis of surfactant, constituting a physiological fibroblast niche (Brody and Kaplan 1983; Endale et al. 2017). Subsequently, coculturing AT2 cells with PDGFR $\alpha^{+}$alveolar fibroblasts (that were found to contain lipid droplets) was shown to enhance their expansion in vitro, suggesting that lipofibroblasts also produce factors that promote AT2 cell proliferation (Barkauskas et al. 2013).

We recently discovered that AT2 celladjacent $P D G F R \alpha^{+}$fibroblasts constitute single-cell Wnt niches for AT2 stem cells, which correspondingly express the canonical Wnt target gene, Axin2, and comprise $\sim 10 \%$ of the AT2 population (Nabhan et al. 2018). The niche fibroblast produces WNT5A (and possibly other Wnts) that signals in a juxtacrine manner to the adjacent AT2 stem cell and typically in an autocrine manner to itself (see Fig. 2, Fibroblast-AT2 panel). Although WNT5A was initially described as a noncanonical Wnt ligand, it was subsequently shown capable of signaling either canonically or noncanonically, depending on which receptor is expressed on the receiving 
N.H. Juul et al.

cell (Mikels and Nusse 2006; van Amerongen et al. 2012). In vitro culture of purified AT2 cells confirmed that WNT5A induces expression of Axin 2 and enables AT2 cell proliferation, reproducing the effects of Wnts that only signal canonically. An important generalized property of WNT proteins is that they are hydrophobic, which restricts their ability to diffuse away from the cell that produces them. Thus, the observed intimate physical association between an AT2 stem cell and adjacent $W n t$-producing $P D G F R \alpha^{+}$fibroblast is essential for maintaining a functional Wnt signaling niche.

Wnt activity in AT2 stem cells appears to have two distinct functions: The first is enabling proliferation, without directly driving it, and the second is inhibiting transdifferentiation into an AT1 cell. Together, these effects of the fibroblast-produced WNT on AT2 cells can be considered as conferring and maintaining "stemness." The role of $W n t$ activity in PDGFR $\alpha^{+}$ fibroblast niche cells that are often also Axin $2^{+}$ has not yet been specifically examined, so it is unknown whether the autocrine Wnt signaling is integral to its AT2 stem cell niche function. As discussed below, alveolar fibroblasts have been shown to also receive signals from nearby cells that modulate their impact on neighboring AT2 stem cells. Overall, considering it also plays a role in supplying lipid substrate for surfactant biogenesis, the AT2 stem cell-associated alveolar niche fibroblast constitutes an integrated "hub" for alveolar physiology.

\section{Two Mechanisms for Actively Restraining AT2} Cell Proliferation

In some tissues, like the intestine, continuous stem cell activity is a feature of normal physiology, whereas in others, like the Drosophila ovariole, stem cell proliferation is tonically inhibited (Losick et al. 2011). Classical radiolabeled thymidine and stathmokinetic experiments revealed that the alveolar epithelium is remarkably quiescent-meaning AT2 stem cells spend most of their time in an inactive state (Spencer and Shorter 1962; Evans and Bils 1969). Recently, two mechanisms for active suppression of AT2 stem cell activity have been identified (see Fig. 2,
"Antiproliferation" symbols). One is mediated by claudin 18 (Cldn18), a tight junction protein whose genetic deletion results in the spontaneous development of multifocal lung adenomas, revealing its role in restraining AT2 cell proliferation (Zhou et al. 2018). The second involves an unidentified factor produced by $B M P R 1 \alpha$ activated alveolar fibroblasts, inferred from studies using experimental unilateral pneumonectomy (Chung et al. 2018). This fibroblastmediated inhibition of AT2 stem cell activation counterbalances the conferral of stemness by the provision of WNT ligand, providing a safeguard against inappropriate proliferation of Axin $2^{+}$ AT2 stem cells. Altogether, the incorporation of multiple distinct anti- and proproliferative cues for AT2 stem cells confers resiliency to their renewal program, because acquired dysfunction of any given one may not be sufficient to disrupt their regulation.

\section{Growth Factors that Drive AT2 Stem Cell Proliferation}

Epidermal growth factor receptor (EGFR) activation is presumed to be an important trigger for AT2 stem cell proliferation in vivo, inferred from its implication as an oncogene for lung adenocarcinoma of AT2 cell origin (Lin et al. 2012; Xu et al. 2012; Desai et al. 2014). The provision of EGF ligands to cultured AT2 cells induces their proliferation, supporting this idea, although the source(s) and identity of the EGF ligand(s) within the AT2 stem cell niche remains unknown. One research group has shown EGFR activation to be important for alveolar regeneration after unilateral pneumonectomy (Ding et al. 2011). They propose a model in which vascular endothelial cells initiate production of $M M P 14$ whose proteolytic activity cleaves HBEGF, liberating the protein to signal to nearby AT2 cells and BASCs (see Fig. 2, Endothelial-BASC/AT2 panel). The cellular source of the HbEGF is unknown, as is the identity and source of the putative endothelial cell-activating signal. It is also not known whether this mechanism is operative during alveolar maintenance or after injury, which presumably activates different signals. Is the autocrine (injury) Wnt program activated in 
"bulk" AT2 cells with pneumonectomy, and are endothelial cells activated by sporadic epithelial cell loss during aging? It will be important to uncover how niche factors regulating independent properties like stemness $(W n t)$ and proliferation (EGFR) are dynamically integrated by AT2 cells during maintenance and after injury.

AT2 cells have also been shown to proliferate when exposed to growth factors activating other receptor families, including insulin-like growth factor receptor (IGFR) and fibroblast growth factor receptor (FGFR). What specific roles these may play in driving AT2 stem cell proliferation in vivo remains to be elucidated. In a spontaneous emphysema mouse model due to a heterozygous mutation in the gene fibrillin 1, which results in dysfunctional collagen, exogenous provision of hepatocyte growth factor (HGF) attenuated airspace enlargement (Calvi et al. 2013). Although the operative mechanism has not been precisely established, genetic deletion in developing lung epithelial cells of the $H G F$ receptor, Met, resulted in airspace enlargement. Together, these findings suggest HGF may play a role in alveolar maintenance by promoting epithelial cell survival or proliferation. The cellular sources of HGF and other candidate growth factors involved in aging and injury have not yet been spatially mapped in vivo in relation to AT2 stem cells.

Regional Aspects of Renewal by AT2 Stem Cells and Implications for Alveolar Diseases

During aging, AT1 cell renewal by AT2 stem cells takes place intermittently in patchy foci located in peripheral (submesothelial) and perivascular alveoli. Upon targeted induction of constitutively active KrasG12D in AT2 cells throughout the lungs, the ones residing in these same regions preferentially activate an adenomatous response (Desai et al. 2014). This enhanced responsiveness implies that AT2 cells in these specific regions are either intrinsically more stem-like, enjoy privileged access to mesothelial and vasculature produced factors that promote Kras-driven proliferation, or both. Submesothelial alveoli are also the site of the greatest AT2 cell proliferation during alveolar regenera- tion after experimental unilateral pneumonectomy and upon refeeding after severe caloric restriction induced emphysema (Massaro et al. 2004; Voswinckel et al. 2004). Intriguingly, peripheral alveoli not only are enriched for agerelated turnover, Kras-responsiveness, and neo-alveolarization but also are the initial site of disease in idiopathic pulmonary fibrosis (IPF) (Martinez et al. 2017). IPF, a disease in which stem cell dysfunction has been implicated, is characterized by distinctive patchy foci of submesothelial alveolar fibrosis and epithelial abnormalities (Carrington et al. 1978). In another disease of elderly smokers, emphysema, patchy foci of alveolar loss may be observed in the lung periphery, referred to as paraseptal emphysema. However, the pathologic alveolar destruction in emphysema is concentrated at the center of the pulmonary lobule, correlating with the initial entry site of inhaled pollutants into the gas exchange compartment via the terminal bronchioles.

\section{INDUCIBLE ALVEOLAR AND AIRWAY NICHE SIGNALS}

A Dynamic Wnt-Niche Switch: Deputizing "Bulk" AT2 Cells for Rapid Alveolar Repair

Despite its slow turnover in health, the alveolar compartment of the lung has a tremendous capacity to rapidly repair acute injury. We found that in the setting of hyperoxic lung injury and moderate lung epithelial ablation, in which the subset of Axin2 $2^{+}$AT2 stem cells may have insufficient capacity, "bulk" (i.e., Axin2-negative) AT2 cells are recruited as progenitors (Nabhan et al. 2018). This broad expansion of the alveolar stem cell pool is associated with induction of Axin2 expression in the surviving AT2 cells. Remarkably, the source of Wnt for injury-activated AT2 cells is the AT2 cells themselves, which rapidly initiate expression of a panel of Wnts that signal in an autocrine manner (see Fig. 2, AT2 intrinsic panel). This switch from a juxtacrine to autocrine Wnt stem cell niche is short-lived, spontaneously resolving once the alveolar epithelium has been repaired. The injury-associated trigger for broad Wnt expression by AT2 cells 
N.H. Juul et al.

is unknown, but induction of Wnts following damage has also been noted in other tissues, so it may be a generalized response to acute injury (Whyte et al. 2012). Interestingly, systemic inhibition of WNT signaling by administration of the porcupine inhibitor C59 results in impaired reepithelialization up to 5 days after injury, by which time much of the alveolar epithelium in control lungs has already been renewed (Nabhan et al. 2018). This notable lack of efficient repair by non-AT2 alveolar progenitors when AT2 cell proliferation has been abrogated by WNT inhibition suggests either that BASCs and LNEP/DASCs were not activated, respond more slowly, also depend on WNT, or are somehow insufficient in the diphtheria toxin-mediated lung epithelial ablation model that was used. Another group independently identified Axin $2^{+}$AT2 cells as making up 20\% of the AT2 cell population and functioning as alveolar stem cells for repair of H1N1 influenza pneumonia (Zacharias et al. 2018).

YAP1 Activity in AT2 Cells Drives Proliferation and Attenuates Alveolar Inflammation

Following unilateral pneumonectomy in rodents, transpulmonary pressures are increased in the remaining lung lobes that undergo compensatory growth with regenerative alveolarization (Hoffman et al. 2010). It was shown almost half a century ago that regeneration was blocked if a wax cast was placed into the evacuated thorax, implicating the alveolar distending force in driving this process (Brody et al. 1978). More recently, it has been reported that AT2 cells and BASCs proliferate postpneumonectomy in response to elevated mechanical tension (see Fig. 2, AT2 intrinsic panel). The Hippo signaling pathway effector, Yes-associated protein 1 (Yap1), was found to be required for AT2 cell proliferation and the process of neo-alveolarization (Liu et al. 2016). In another context, Yap1 was implicated as the driver of spontaneous AT2 cell proliferation resulting from genetic deletion of Cldn18, caused by loss of sequestration of YAP1 protein at the cell junction (see Fig. 2, AT2 intrinsic panel) (Zhou et al. 2018). As discussed below, Yap1 and Taz activity in AT2 cells has also been shown to facilitate alveolar repair by suppressing inflammation in the setting of bacterial pneumonia (LaCanna et al. 2019). Yap1 activity in AT2 cells has thus been implicated as an important promoter of alveolar repair by two different mechanisms, driving AT2 stem cell proliferation and attenuating the immune response after injury.

\section{Resident and Recruited Immune Cells Modulate AT2 Cell Activation in Repair and Regeneration}

There is evidence that immune cells have important noncanonical effects on alveolar epithelial stem cell activity, and sessile macrophages have even been reported to share direct physical connections with AT2 cells (Westphalen et al. 2014). During homeostasis, in addition to patrolling for pathogens, alveolar macrophages cooperate with AT2 cells in surfactant homeostasis. During infection, both AT2 cells and macrophages produce factors that recruit immune cells like neutrophils and T-effector cells to the site of injury. After cessation of recruitment, neutrophils and some macrophages undergo apoptosis, although the remaining cells modulate alveolar epithelial restoration (Aggarwal et al. 2014). Some genetic studies have examined communication between AT2 and immune cells in vivo during infection. In one recent report, conditional deletion of Yap1 and Taz in AT2 cells in the setting of bacterial pneumonia resulted in excessive proinflammatory cytokine production, resulting in delayed alveolar repair (LaCanna et al. 2019).

In other settings, immune cells have been found to facilitate, rather than impair, alveolar regeneration (see Fig. 2, Immune-AT2 panel). One such mechanism following experimental unilateral pneumonectomy has been well-delineated. Here, secretion of CCL 2 by AT2 cells recruits $C C R 2^{+}$monocytes to the lung where they mature and become alternately activated via innate lymphoid cell 2 (ILC2)-produced interleukin (IL)-13, whereupon they promote AT2 cell proliferation through an unknown mediator (Lechner et al. 2017). In another study, $I L-1 \beta$ and tumor necrosis factor $\alpha(T N F-\alpha)$ were in- 
duced by H1N1 influenza pneumonia and found to signal directly to AT2 cells to promote their proliferation (Katsura et al. 2019). Future studies will be important for dissecting the particular immune cell populations and specific signals that mediate these at times opposing effects on alveolar repair in infectious and noninfectious states.

Summoning "Reserve" Alveolar Progenitors and an Airway Smooth Muscle FGF Niche

How are progenitor cells residing in airways able to remotely sense and respond to cell loss in the alveolar compartment? One possibility is that they receive activating signals via an intermediary-for instance, mobile immune cells broadly infiltrating an injured lung. In this regard, it is interesting that monocytes are recruited to the lung following unilateral pneumonectomy, despite the absence of injured or dying cells. Another possibility is that the injury directly involves the airway progenitor cell microenvironment, which would explain why reserve progenitors have been found to be activated when the damage is not limited to the alveolar compartment-for instance, with bleomycin or H1N1 influenza. To properly generate new alveolar cells, LNEP/DASC and BASC cell progeny must migrate distally along the bronchiolar surface to reach the gas exchange compartment. This ingression might be facilitated by damaged terminal airways along which progenitors could move without having to dynamically remodel epithelial cell-cell junctions. Loss of epithelial integrity might also derepress "reserve" airway progenitors if, like AT2 cells, their proliferation is inhibited by sequestration of proteins like YAP1 at cell-cell junctions. It currently remains unknown which, if any, of these potential mechanisms for alerting the airway "reserve" progenitors to alveolar perturbations is operative in vivo.

For LNEP/DASC and BASC cells, it has recently been shown that with severe acute injury, airway smooth muscle cells produce FGF10, which induces proliferation and migration of nearby epithelial progenitors (see Fig. 2, SMCLNEP/BASC panel). This study found that de- letion of either FGF10 in smooth muscle cells or its receptor, FGFR2b, in airway epithelial cells markedly reduced the number of $K r t 5^{+}$LNEP/ DASCs found in the terminal bronchioles and alveoli following bleomycin administration (Yuan et al. 2019). There was also an accompanying lack of AT2 cell differentiation, although whether this resulted from a failure of the cells to reach the alveoli or directly from the absence of FGFR activation is unclear.

The niche cells and signals that control LNEP/DASC and BASC stemness in vivo remain unknown. More proximally in the trachea, however, FGF signaling has been shown to maintain basal cell "stemness." One research group reported that haploinsufficiency of FGFR2 in $K r t 5^{+}$basal cells resulted in stem cell depletion caused by differentiation without selfrenewal (Balasooriya et al. 2017). Another study identified thrombospondin 1 (TSP1) produced by vascular endothelial cells in a BMP4-dependent manner as favoring BASC differentiation toward an AT2 cell phenotype, as opposed to bronchiolar lineages, on airway and alveolar injury (see Fig. 2, Endothelial-BASC/AT2 panel; Lee et al. 2014).

\section{LEAVING THE NICHE: SEQUENTIAL SIGNALING ORCHESTRATES REGENERATIVE OUTCOMES}

WNTS not only endow AT2 cells with proliferative capacity but also impact their differentiation status. Either deletion of $\beta$-catenin in vivo or inhibition of Wnt in vitro triggers AT1 transdifferentiation of a subset of AT2 cells, presumably Axin $2^{+}$stem cells because of abrogation of $W n t$ signaling. In contrast, experimental induction of stabilized $\beta$-catenin in AT2 cells does not drive proliferation but blocks AT1 transdifferentiation (Nabhan et al. 2018). In vivo, following physiologic Axin2 ${ }^{+}$AT2 stem cell duplication, one daughter cell is by default physically excluded from proximity to the WNT-producing fibroblast niche. Yet, lineage tracing of Axin2 ${ }^{+}$ AT2 cells reveals that they generate both AT1 and AT2 cells, indicating that abrogation of active Wnt signaling is not always sufficient to drive AT1 transdifferentiation. Indeed, a recent 
N.H. Juul et al.

report using bleomycin injury and AT2 cell spheroid culture found that the Hippo signaling pathway effector, Taz, is required for AT2 to AT1 cell conversion (Sun et al. 2019).

The temporal sequence and hierarchy of signals mediating proper execution of the proliferation, migration, and differentiation steps of the alveolar progenitor programs are beginning to be elucidated (see Fig. 3). For AT2 cells, it appears multistep signaling via $B M P R 1 \alpha$ is important for generating new AT1 cells. BMP signaling is initially down-regulated in AT2 cells during proliferation, and then it increases during AT1 cell transdifferentiation (Chung et al.
2018). Notch signaling, in contrast, is induced during AT2 cell activation and then is downregulated during conversion into an AT1 cell (Finn et al. 2019). Interestingly, Notch is also important for LNEP/DASC activation, but subsequent AT2 cell differentiation is promoted by inhibition of Notch and induction of Wnt signaling (Vaughan et al. 2015). Thus, down-regulation of Notch can be associated with either loss or acquisition of the AT2 cell phenotype, depending on context. This divergent response to the identical signal suggests a hysteresis, in which the differentiation outcome of a lung progenitor is affected by preceding steps in the cell's
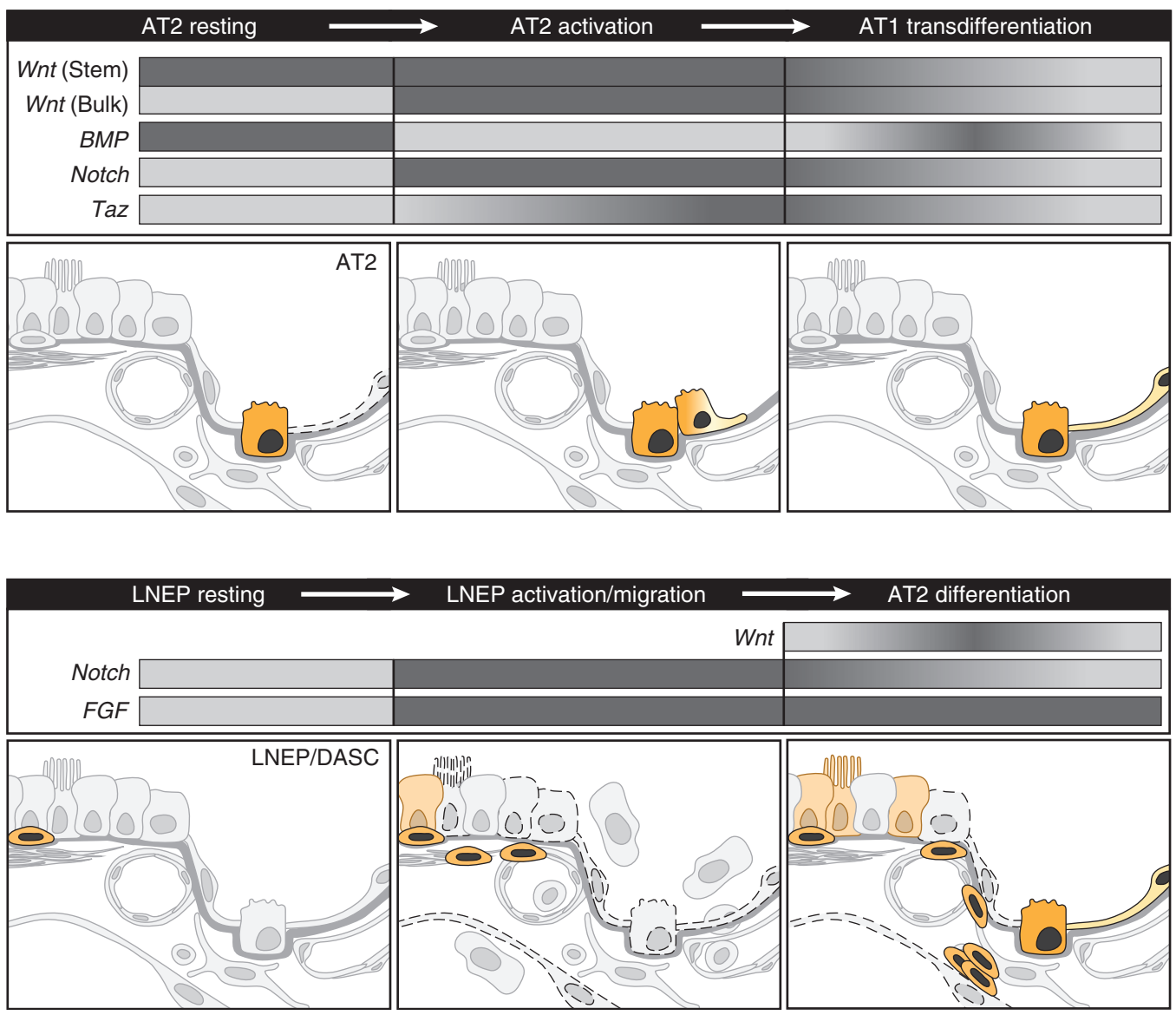

Figure 3. Dynamic signaling in alveolar stem cells that sequentially drive proliferation and differentiation. Signaling pathway activity (dark shade indicates active) in alveolar type II (AT2) cells (top) and LNEP/DASC cells (bottom) during sequential steps of their regenerative programs. "Stem" indicates Axin2 ${ }^{+}$and "Bulk" indicates Axin2 ${ }^{-}$AT2 cells. Dashed outlines represent dying cells. 
history. Additional work is needed to fully unravel the extrinsic signals that orchestrate the proper sequence and timing of each step in the distinct alveolar regenerative programs.

\section{AT2 STEM CELL NICHES AND SIGNALS IN LUNG ADENOCARCINOMA}

The appreciation that every adenocarcinoma harbors molecularly heterogeneous tumor cells ushered in an experimental era of prospectively isolating distinct epithelial subsets and comparing their cancer-propagating activity when transplanted into immunocompromised mice. This approach is based on the idea that the most efficient subset may be selectively endowed with stem cell-like capacity that makes it essential for continued growth of the primary tumor and, thus, an attractive therapeutic target (Kreso and Dick 2014). However, a recent study showed in a mouse model of colon cancer that targeted elimination of a tumor propagating subset in vivo resulted in its replenishment by other cells that altered their phenotype, along with a failure to eradicate the primary tumor (de Sousa e Melo et al. 2017). This result illustrates how cells in the tumor microenvironment can exert profound control over epithelial cells in cancer, as they do on stem cells in normal physiology. Although some niche interactions in cancer may mediate susceptibility or resistance to elimination by infiltrating immune cells, others recapitulate their normal physiologic function in the specific epithelial cell type that generated the tumor.

In the lung, AT2 cells are susceptible to adenomatous transformation by constitutive activation of EGFR or the downstream Kras pathway, which appears to normally drive their proliferation in health. Intriguingly, Wnt activity in adenocarcinoma is important for cancer progression even though Kras is constitutively active, suggesting that its physiologic role in conferring proliferative competence and blocking AT1 transdifferentiation continues to be required (Tammela et al. 2017). Another example is M2 polarized alveolar macrophages, which have long been known to promote lung adenoma progression, mirroring their physiologic effect in enhancing regenerative neo-alveolarization by AT2 cells after pneumonectomy (Zaynagetdinov et al. 2011). Recently, a study showed attenuation of Kras-driven lung adenoma growth with inhibition of $\gamma \delta \mathrm{T}$-cell activation, implicating a different immune-mediated pathway that promotes epithelial cell proliferation (Jin et al. 2019). As with the M2 macrophage effect, $\gamma \delta$ T cells augment proliferation in epithelial cells that already have constitutive Kras activity, suggesting they drive proliferation through a distinct (non-Kras) pathway and/or somehow augment "stemness." Figure 4 schematizes what is currently known about the lung adenocarcinoma "tumor niche" interactions. Future work is needed to identify and characterize additional niche cells and signals that regulate healthy alveolar regeneration and may be conserved in cancer, including interactions mediating more complex processes like neoangiogenesis.

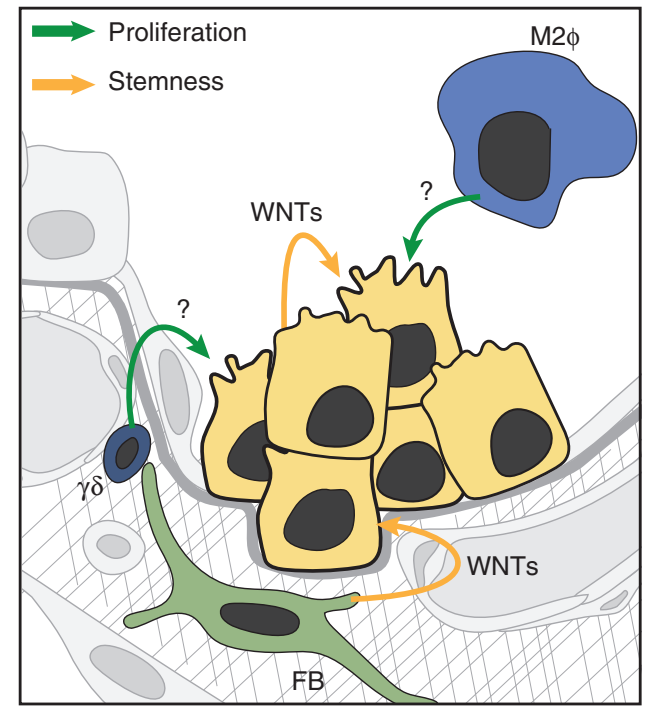

Figure 4. Lung adenocarcinoma "tumor niche." Tumor-associated cells and the signals they produce that have been shown to confer proliferative competence (yellow arrows) and augment proliferation (green arrow) of epithelial tumor cells in Kras-driven lung adenocarcinoma. (FB) Fibroblast, $(\gamma \delta) \gamma \delta \mathrm{T}$ cell, (M2 $\Phi)$ alternately activated macrophage, (?) unknown signal(s). 
N.H. Juul et al.

\section{CONCLUDING REMARKS}

Mouse genetics has enabled the identification of distinct niche cells and signals that influence alveolar stem cell behavior in vivo under different conditions. One regulatory mode involves tonic provision of a single factor to maintain a specific state, exemplified by Wnt activity in AT2 stem cells mediating proliferative competency and differentiation inhibition. More complex behaviors involve the orchestrated execution of discrete stem cell activities-for instance, abrogation of quiescence, proliferation, migration, and differentiation of LNEP/DASCs after severe lung injury. Such stereotyped stem cell "programs" necessitate precise coordination of signaling arcs involving multiple pathways and may involve hysteresis, making it difficult to reconcile apparently disparate effects of the same factor in different physiologic contexts. Integrating diverse experimental findings will bring us closer to a holistic understanding of how alveolar stem cells are regulated in vivo. A critical next step is to translate these findings from mouse into human lung, which has been facilitated by the development of organoid assays, cocultures, and intact lung slice cultures (Nikolić and Rawlins 2017). The latter approach is particularly important because it enables manipulation of alveolar stem cell signaling within the context of a mostly intact niche, essential for testing candidate therapeutics (Lehmann et al. 2017). The comprehensive identification of the diverse signals and their effects on human alveolar stem cells and how they are specifically perturbed in disease will ultimately provide a blueprint for developing new regenerative and anticancer therapies.

\section{ACKNOWLEDGMENTS}

This work was supported by National Institutes of Health (NIH) 5R01HL14254902 (T.J.D.), the Virginia and D.K. Ludwig Fund for Cancer Research (T.J.D.), Stanford School of Medicine Dean's Postdoctoral Fellowship Award (N.H.J.), 1T32HL129970-01A1, 5T32HL12997003 (N.H.J.), 1F32HL147417 (N.H.J), and 5T32G M119995-02 (C.A.S). T.J.D. is the Woods Fam- ily Faculty Scholar in Pediatric Translational Medicine of the Stanford Maternal \& Child Health Research Institute.

\section{REFERENCES}

Adamson IY, Bowden DH. 1974. The type 2 cell as progenitor of alveolar epithelial regeneration. A cytodynamic study in mice after exposure to oxygen. Lab Invest 30: 35-42.

Aggarwal NR, King LS, D’Alessio FR. 2014. Diverse macrophage populations mediate acute lung inflammation and resolution. Am J Physiol Lung Cell Mol Physiol 306: L709L725. doi:10.1152/ajplung.00341.2013

Balasooriya GI, Goschorska M, Piddini E, Rawlins EL. 2017. FGFR2 is required for airway basal cell self-renewal and terminal differentiation. Development 144: 1600-1606. doi:10.1242/dev.135681

Barkauskas CE, Cronce MJ, Rackley CR, Bowie EJ, Keene DR, Stripp BR, Randell SH, Noble PW, Hogan BLM. 2013. Type 2 alveolar cells are stem cells in adult lung. $J$ Clin Invest 123: 3025-3036. doi:10.1172/JCI68782

Bertalanffy FD, Leblond CP. 1955. Structure of respiratory tissue. Lancet 266: 1365-1368. doi:10.1016/S0140-6736 (55) $93164-0$

Brody JS, Kaplan NB. 1983. Proliferation of alveolar interstitial cells during postnatal lung growth: evidence for two distinct populations of pulmonary fibroblasts. Am Rev Respir Dis 127: 763-770.

Brody JS, Burki R, Kaplan N. 1978. Deoxyribonucleic acid synthesis in lung cells during compensatory lung growth after pneumonectomy. Am Rev Respir Dis 117: 307-316.

Calvi C, Podowski M, Lopez-Mercado A, Metzger S, Misono K, Malinina A, Dikeman D, Poonyagariyon H, Ynalvez L, Derakhshandeh R, et al. 2013. Hepatocyte growth factor, a determinant of airspace homeostasis in the murine lung. PLoS Genet 9: e1003228. doi:10.1371/journal.pgen.100 3228

Carrington CB, Gaensler EA, Coutu RE, FitzGerald MX, Gupta RG. 1978. Natural history and treated course of usual and desquamative interstitial pneumonia. New Engl J Med 298: 801-809. doi:10.1056/NEJM 197804132981501

Chapman HA, Li X, Alexander JP, Brumwell A, Lorizio W, Tan K, Sonnenberg A, Wei Y, Vu TH. 2011. Integrin $\alpha 6 \beta 4$ identifies an adult distal lung epithelial population with regenerative potential in mice. J Clin Invest 121: 28552862. doi:10.1172/JCI57673

Chung MI, Bujnis M, Barkauskas CE, Kobayashi Y, Hogan BLM. 2018. Niche-mediated BMP/SMAD signaling regulates lung alveolar stem cell proliferation and differentiation. Development 145: dev163014. doi:10.1242/dev.16 3014

Crapo JD, Young SL, Fram EK, Pinkerton KE, Barry BE, Crapo RO. 1983. Morphometric characteristics of cells in the alveolar region of mammalian lungs. Am Rev Respir Dis 128: S42-S46.

Desai TJ, Brownfield DG, Krasnow MA. 2014. Alveolar progenitor and stem cells in lung development, renewal and cancer. Nature 507: 190-194. doi:10.1038/nature12930 
de Sousa e Melo F, Kurtova AV, Harnoss JM, Kljavin N, Hoeck JD, Hung J, Anderson JE, Storm EE, Modrusan Z, Koeppen H, et al. 2017. A distinct role for $\mathrm{Lgr5}^{+}$stem cells in primary and metastatic colon cancer. Nature 543: 676-680. doi:10.1038/nature21713

Ding BS, Nolan DJ, Guo P, Babazadeh AO, Cao Z, Rosenwaks Z, Crystal RG, Simons M, Sato TN, Worgall S, et al. 2011. Endothelial-derived angiocrine signals induce and sustain regenerative lung alveolarization. Cell 147: 539553. doi:10.1016/j.cell.2011.10.003

Endale M, Ahlfeld S, Bao E, Chen X, Green J, Bess Z, Weirauch MT, Xu Y, Perl AK. 2017. Temporal, spatial, and phenotypical changes of PDGFR $\alpha$ expressing fibroblasts during late lung development. Dev Biol 425: 161-175. doi:10.1016/j.ydbio.2017.03.020

Evans MJ, Bils RF. 1969. Identification of cells labeled with tritiated thymidine in the pulmonary alveolar walls of the mouse. Am Rev Respir Dis 100: 372-378. doi:10.1164/ arrd.1969.100.3.372

Evans MJ, Cabral LJ, Stephens RJ, Freeman G. 1973. Renewal of alveolar epithelium in the rat following exposure to $\mathrm{NO}_{2}$. Am J Pathol 70: 175-198.

Finn J, Sottoriva K, Pajcini KV, Kitajewski JK, Chen C, Zhang W, Malik AB, Liu Y. 2019. Dlk1-mediated temporal regulation of Notch signaling is required for differentiation of alveolar type II to type I cells during repair. Cell Rep 26: 2942-2954.e5. doi:10.1016/j.celrep.2019.02.046

Haies DM, Gil J, Weibel ER. 1981. Morphometric study of rat lung cells. I: Numerical and dimensional characteristics of parenchymal cell population. Am Rev Respir Dis 123: $533-541$.

Hoffman AM, Shifren A, Mazan MR, Gruntman AM, Lascola KM, Nolen-Walston RD, Kim CF, Tsai L, Pierce RA, Mecham RP, et al. 2010. Matrix modulation of compensatory lung regrowth and progenitor cell proliferation in mice. Am J Physiol Lung Cell Mol Physiol 298: L158-L168. doi:10.1152/ajplung.90594.2008

Jain R, Barkauskas CE, Takeda N, Bowie EJ, Aghajanian $\mathrm{H}$, Wang Q, Padmanabhan A, Manderfield LJ, Gupta M, Li D, et al. 2015. Plasticity of $\mathrm{Hopx}^{+}$type I alveolar cells to regenerate type II cells in the lung. Nat Commun 6: 6727. doi:10.1038/ncomms 7727

Jin C, Lagoudas GK, Zhao C, Bullman S, Bhutkar A, Hu B, Ameh S, Sandel D, Liang XS, Mazzilli S, et al. 2019. Commensal microbiota promote lung cancer development via $\gamma \delta$ T cells. Cell 176: 998-1013.e16. doi:10.1016/j.cell.2018 .12 .040

Kapanci Y, Weibel ER, Kaplan HP, Robinson FR. 1969. Pathogenesis and reversibility of the pulmonary lesions of oxygen toxicity in monkeys. II: Ultrastructural and morphometric studies. Lab Invest 20: 101-118.

Katsura H, Kobayashi Y, Tata PR, Hogan BLM. 2019. IL-1 and TNF $\alpha$ contribute to the inflammatory niche to enhance alveolar regeneration. Stem Cell Reports 12: 657666. doi:10.1016/j.stemcr.2019.02.013

Kim CF, Jackson EL, Woolfenden AE, Lawrence S, Babar I, Vogel S, Crowley D, Bronson RT, Jacks T. 2005. Identification of bronchioalveolar stem cells in normal lung and lung cancer. Cell 121: 823-835. doi:10.1016/j.cell.2005.03 .032
Kreso A, Dick JE. 2014. Evolution of the cancer stem cell model. Cell Stem Cell 14: 275-291. doi:10.1016/j.stem .2014 .02 .006

Kumar PA, Hu Y, Yamamoto Y, Hoe NB, Wei TS, Mu D, Sun Y, Joo LS, Dagher R, Zielonka EM, et al. 2011. Distal airway stem cells yield alveoli in vitro and during lung regeneration following H1N1 influenza infection. Cell 147: 525-538. doi:10.1016/j.cell.2011.10.001

LaCanna R, Liccardo D, Zhang P, Tragesser L, Wang Y, Cao T, Chapman HA, Morrisey EE, Shen H, Koch WJ, et al. 2019. Yap/Taz regulate alveolar regeneration and resolution of lung inflammation. J Clin Invest 129: 2107-2122. doi:10.1172/JCI125014

Lechner AJ, Driver IH, Lee J, Conroy CM, Nagle A, Locksley RM, Rock JR. 2017. Recruited monocytes and type $2 \mathrm{im}$ munity promote lung regeneration following pneumonectomy. Cell Stem Cell 21: 120-134.e7. doi:10.1016/j .stem.2017.03.024

Lee JH, Bhang DH, Beede A, Huang TL, Stripp BR, Bloch KD, Wagers AJ, Tseng YH, Ryeom S, Kim CF. 2014. Lung stem cell differentiation in mice directed by endothelial cells via a BMP4-NFATc1-thrombospondin-1 axis. Cell 156: 440-455. doi:10.1016/j.cell.2013.12.039

Lehmann M, Korfei M, Mutze K, Klee S, Skronska-Wasek W, Alsafadi HN, Ota C, Costa R, Schiller HB, Lindner M, et al. 2017. Senolytic drugs target alveolar epithelial cell function and attenuate experimental lung fibrosis ex vivo. Eur Respir J 50: 1602367. doi:10.1183/13993003.023672016

Lin C, Song H, Huang C, Yao E, Gacayan R, Xu SM, Chuang PT. 2012. Alveolar type II cells possess the capability of initiating lung tumor development. PLoS ONE 7: e53817. doi:10.1371/journal.pone.0053817

Liu Z, Wu H, Jiang K, Wang Y, Zhang W, Chu Q, Li J, Huang H, Cai T, Ji H, et al. 2016. MAPK-mediated YAP activation controls mechanical-tension-induced pulmonary alveolar regeneration. Cell Rep 16: 1810-1819. doi:10.1016/ j.celrep.2016.07.020

Liu Q, Liu K, Cui G, Huang X, Yao S, Guo W, Qin Z, Li Y, Yang $\mathrm{R}, \mathrm{Pu} \mathrm{W}$, et al. 2019. Lung regeneration by multipotent stem cells residing at the bronchioalveolar-duct junction. Nat Genet 51: 728-738. doi:10.1038/s41588019-0346-6

Logan CY, Desai TJ. 2015. Keeping it together: pulmonary alveoli are maintained by a hierarchy of cellular programs. Bioessays 37: 1028-1037. doi:10.1002/bies.201500031

Losick VP, Morris LX, Fox DT, Spradling A. 2011. Drosophila stem cell niches: a decade of discovery suggests a unified view of stem cell regulation. Dev Cell 21: 159-171. doi:10.1016/j.devcel.2011.06.018

Low FN. 1953. The pulmonary alveolar epithelium of laboratory mammals and man. Anat Rec 117: 241-263. doi:10 $.1002 /$ ar. 1091170208

Martinez FJ, Collard HR, Pardo A, Raghu G, Richeldi L, Selman M, Swigris JJ, Taniguchi H, Wells AU. 2017. Idiopathic pulmonary fibrosis. Nat Rev Dis Primers 3: 17074. doi:10.1038/nrdp.2017.74

Massaro D, Massaro GD, Baras A, Hoffman EP, Clerch LB. 2004. Calorie-related rapid onset of alveolar loss, regeneration, and changes in mouse lung gene expression. Am J Physiol Lung Cell Mol Physiol 286: L896-L906. doi:10 .1152/ajplung.00333.2003 
N.H. Juul et al.

Mikels AJ, Nusse R. 2006. Purified Wnt5a protein activates or inhibits $\beta$-catenin-TCF signaling depending on receptor context. PLoS Biol 4: e115. doi:10.1371/journal.pbio .0040115

Nabhan A, Brownfield DG, Harbury PB, Krasnow MA, Desai TJ. 2018. Single-cell Wnt signaling niches maintain stemness of alveolar type 2 cells. Science 359: 1118-1123. doi:10.1126/science.aam6603

Nikolić MZ, Rawlins EL. 2017. Lung organoids and their use to study cell-cell interaction. Curr Pathobiol Rep 5: $223-$ 231. doi:10.1007/s40139-017-0137-7

Rawlins EL, Okubo T, Xue Y, Brass DM, Auten RL, Hasegawa H, Wang F, Hogan BLM. 2009. The role of Scgbla1 ${ }^{+}$ Clara cells in the long-term maintenance and repair of lung airway, but not alveolar, epithelium. Cell Stem Cell 4: 525-534. doi:10.1016/j.stem.2009.04.002

Ray S, Chiba N, Yao C, Guan X, McConnell AM, Brockway B, Que L, McQualter JL, Stripp BR. 2016. Rare SOX2 airway progenitor cells generate $\mathrm{KRT}^{+}$cells that repopulate damaged alveolar parenchyma following influenza virus infection. Stem Cell Reports 7: 817-825. doi:10 $.1016 / j . s t e m c r .2016 .09 .010$

Rock JR, Onaitis MW, Rawlins EL, Lu Y, Clark CP, Xue Y, Randell SH, Hogan BLM. 2009. Basal cells as stem cells of the mouse trachea and human airway epithelium. Proc Natl Acad Sci 106: 12771-12775. doi:10.1073/pnas .0906850106

Sirianni FE, Chu FS, Walker DC. 2003. Human alveolar wall fibroblasts directly link epithelial type 2 cells to capillary endothelium. Am J Resp Crit Care 168: 1532-1537. doi:10 .1164/rccm.200303-371OC

Spencer H, Shorter RG. 1962. Cell turnover in pulmonary tissues. Nature 194: 880. doi:10.1038/194880a0

Sun T, Huang Z, Zhang H, Posner C, Jia G, Ramalingam TR, Xu M, Brightbill H, Egen JG, Dey A, et al. 2019. TAZ is required for lung alveolar epithelial cell differentiation after injury. JCI Insight 5: 128674. doi:10.1172/jci .insight. 128674

Tammela T, Sanchez-Rivera FJ, Cetinbas NM, Wu K, Joshi NS, Helenius K, Park Y, Azimi R, Kerper NR, Wesselhoeft RA, et al. 2017. A Wnt-producing niche drives proliferative potential and progression in lung adenocarcinoma. Nature 545: 355-359. doi:10.1038/nature22334

van Amerongen R, Fuerer C, Mizutani M, Nusse R. 2012. Wnt5a can both activate and repress Wnt/ $\beta$-catenin signaling during mouse embryonic development. Dev Biol 369: 101-114. doi:10.1016/j.ydbio.2012.06.020

Vaughan AE, Brumwell AN, Xi Y, Gotts JE, Brownfield DG, Treutlein B, Tan K, Tan V, Liu FC, Looney MR, et al. 2015.
Lineage-negative progenitors mobilize to regenerate lung epithelium after major injury. Nature 517: 621-625. doi:10.1038/nature14112

Voswinckel R, Motejl V, Fehrenbach A, Wegmann M, Mehling T, Fehrenbach H, Seeger W. 2004. Characterisation of post-pneumonectomy lung growth in adult mice. Eur Respir J 24: 524-532. doi:10.1183/09031936.04.10004904

Westphalen K, Gusarova GA, Islam MN, Subramanian M, Cohen TS, Prince AS, Bhattacharya J. 2014. Sessile alveolar macrophages communicate with alveolar epithelium to modulate immunity. Nature 506: 503-506. doi:10 1038/nature12902

Whyte JL, Smith AA, Helms JA. 2012. Wnt signaling and injury repair. Cold Spring Harb Perspect Biol 4: a008078. doi:10.1101/cshperspect.a008078

Xu X, Rock JR, Lu Y, Futtner C, Schwab B, Guinney J, Hogan BL, Onaitis MW. 2012. Evidence for type II cells as cells of origin of K-Ras-induced distal lung adenocarcinoma. Proc Natl Acad Sci 109: 4910-4915. doi:10.1073/pnas .1112499109

Yuan T, Volckaert T, Redente EF, Hopkins S, Klinkhammer K, Wasnick R, Chao CM, Yuan J, Zhang JS, Yao C, et al. 2019. FGF10-FGFR2B signaling generates basal cells and drives alveolar epithelial regeneration by bronchial epithelial stem cells after lung injury. Stem Cell Reports 12: 1041-1055. doi:10.1016/j.stemcr.2019.04.003

Zacharias WJ, Frank DB, Zepp JA, Morley MP, Alkhaleel FA, Kong J, Zhou S, Cantu E, Morrisey EE. 2018. Regeneration of the lung alveolus by an evolutionarily conserved epithelial progenitor. Nature 555: 251-255. doi:10.1038/ nature25786

Zaynagetdinov R, Sherrill TP, Polosukhin VV, Han W, Ausborn JA, McLoed AG, McMahon FB, Gleaves LA, Degryse AL, Stathopoulos GT, et al. 2011. A critical role for macrophages in promotion of urethane-induced lung carcinogenesis. J Immunol 187: 5703-5711. doi:10 .4049/jimmunol.1100558

Zhou B, Flodby P, Luo J, Castillo DR, Liu Y, Yu FX, McConnell A, Varghese B, Li G, Chimge NO, et al. 2018. Claudin-18-mediated YAP activity regulates lung stem and progenitor cell homeostasis and tumorigenesis. J Clin Invest 128: 970-984. doi:10.1172/JCI90429

Zuo W, Zhang T, Wu DZ, Guan SP, Liew AA, Yamamoto Y, Wang X, Lim SJ, Vincent M, Lessard M, et al. 2015. p $63^{+} \mathrm{Krt5}^{+}$distal airway stem cells are essential for lung regeneration. Nature 517: 616-620. doi:10.1038/nature 13903 


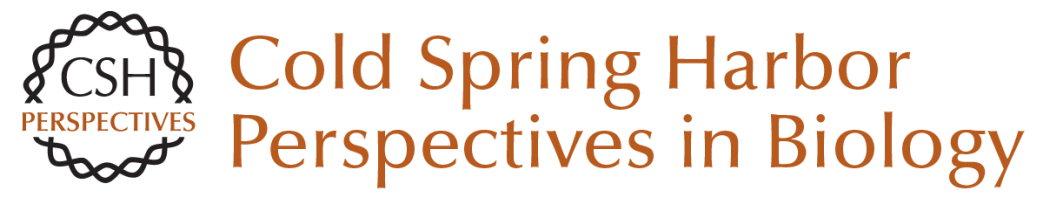

\section{Niche Cells and Signals that Regulate Lung Alveolar Stem Cells In Vivo}

Nicholas H. Juul, Courtney A. Stockman and Tushar J. Desai

Cold Spring Harb Perspect Biol 2020; doi: 10.1101/cshperspect.a035717 originally published online March 16, 2020

\section{Subject Collection Stem Cells: From Biological Principles to Regenerative Medicine}

A Stem Cell Approach to Cure Type 1 Diabetes Aharon Helman and Douglas A. Melton

Niche Cells and Signals that Regulate Lung Alveolar Stem Cells In Vivo Nicholas H. Juul, Courtney A. Stockman and Tushar J. Desai

Stem Cell DNA Damage and Genome Mutation in the Context of Aging and Cancer Initiation Lara Al zouabi and Allison J. Bardin

Capturing Stem Cell Behavior Using Intravital and Live Cell Microscopy

Arianna Fumagalli, Lotte Bruens, Colinda L.G.J. Scheele, et al.

Brain Organoids: Human Neurodevelopment in a Dish

Silvia Benito-Kwiecinski and Madeline A. Lancaster

Advancing Stem Cell Research through

Multimodal Single-Cell Analysis

Iwo Kucinski and Berthold Gottgens

Tracing the Dynamics of Stem Cell Fate

Lemonia Chatzeli and Benjamin D. Simons
Mouse Spermatogenesis Reflects the Unity and Diversity of Tissue Stem Cell Niche Systems Shosei Yoshida

A Synthesis Concerning Conservation and Divergence of Cell Types across Epithelia Daniel T. Montoro, Adam L. Haber, Jennifer E. Rood, et al.

Discovering New Progenitor Cell Populations through Lineage Tracing and In Vivo Imaging Rudra Nayan Das and Karina Yaniv

Next-Generation Biomaterials for Culture and Manipulation of Stem Cells

Koichiro Uto, Christopher K. Arakawa and Cole A. DeForest

Development of Stem Cell Therapies for Retinal Degeneration Emma L. West, Joana Ribeiro and Robin R. Ali

Direct Lineage Reprogramming: Harnessing Cell

Plasticity between Liver and Pancreas Silvia Ruzittu, David Willnow and Francesca M. Spagnoli

Modeling Brain Disorders Using Induced

Pluripotent Stem Cells Krishna C. Vadodaria, Jeffrey R. Jones, Sara Linker, et al.

For additional articles in this collection, see http://cshperspectives.cshlp.org/cgi/collection/

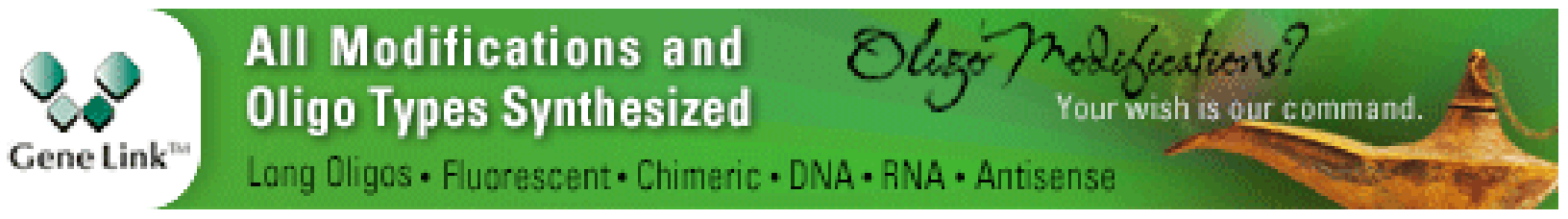


Toward Combined Cell and Gene Therapy for

\section{Genodermatoses}

Laura De Rosa, Maria Carmela Latella, Alessia Secone Seconetti, et al.

\section{Specification of the First Mammalian Cell \\ Lineages In Vivo and In Vitro \\ Melanie D. White and Nicolas Plachta}

For additional articles in this collection, see http://cshperspectives.cshlp.org/cgi/collection/

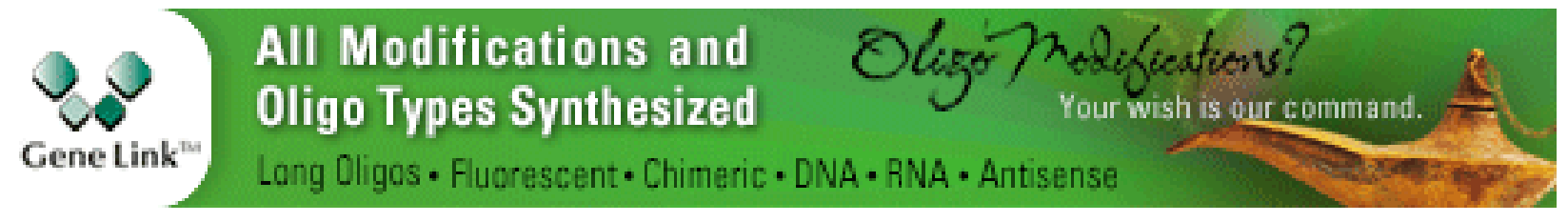

Copyright @ 2020 Cold Spring Harbor Laboratory Press; all rights reserved 